\title{
REVUE
}

\section{LA PASTEURISATION DU LAIT PAR CHAUFFAGE PAR HAUTE FRÉQUENCE}

\author{
par \\ G. GÉNIN \\ Ingénieur E.P.C.I.
}

La seconde guerre mondiale, qui a modifié profondément de nombreuses techniques industrielles, a apporté, en particulier dans les méthodes de chauffage et de séchage, de profondes transformations à la suite de la mise au point de méthodes de chauffage par rayons infra-rouges et par haute fréquence.

Ici même, M. Deribèré [1] a décrit une méthode de séchage de la caséine par rayonnement infra-rouge. Ce procédé de chauffage a été étendu à de nombreuses branches de l'industrie alimentaire et nous citerons par exemple le séchage du poisson [2], la dessication de nombreux aliments et en particulier les légumes, la fabrication de la pénicilline [3], etc.

D'apparition encore plus récente, le séchage diélectrique qui repose sur l'emploi d'un champ électrique à très haute fréquence a été également utilisé pour le séchage des légumes [4] en partieulier betteraves, carottes, choux, oignons et pour la fabrication de ee que l'on appelle aujourd'hui les aliments déshydratés. L'expérience a en effet montré que cette méthode de séchage permettait de gagner un temps considérable et que grâce à elle, on parvenait à éliminer les dernières traces d'humidité contenues dans un produit alimentaire, sans altérer ce dernier.

Ce procédé a également été utilisé pour la pasteurisation dū lait et c'est cette application particulière que nous allons décrire maintenant, en en montrant les avantages par rapport aux procédés anciens de pasteurisation. Toutefois auparavant, nous aurons à décrire, tout au moins succinctement, ce qu'est le chauffage par haute fréquence, ou encore le chauffage électronique, suivant la terminologie américaine.

Chacun connaît l'utilisation des courants à haute fréquence qui est faite dans le chauffage par induction et qui consiste à soumettre un corps conducteur à un champ magnétique variable. Ce procédé de chauffage est très employé en métallurgie, en particulier dans la métallurgie des métaux légers et il a également trouvé dans la construction mécanique de très importants débouchés dans tous les cas où l'on cherche à réaliser un chauffage extrêmement rapide et très localisé. 
On sait également que le chauffage d'un corps conducteur placé dans un ehamp magnétique est dû à l'apparition dans ce corps conducteur de courants induits, appelés encore courants de Foucault, et parfois également courants tourbillonnaires et que le passage de ces courants dans le métal, par suite de l'effet Joule, entraîne l'échauffement de celui-ci.

Lorsqu'en 1937, deux savants français, Leduc et Dufour constatant les difficultés que l'on rencontrait lorsque le problème se posait du chauffage et de la vulcanisation d'objets en caoutchoue de forte épaisseur, songèrent à utiliser ce mode de chauffage, ils furent donc conduits à incorporer dans le caoutchouc des matières conductrices, par exemple des particules finement divisées de carbone, afin de permettre la circulation de ces courants dans une masse non conductrice, comme l'est le caoutchoue. Mais ces premiers essais ne furent pas couronnés de sucès, car pour obtenir une élévation suffisante de température, il était nécessaire d'incorporer dans le mélange des proportions élévées de substances conductrices qui en modifiaient considérablement les caractéristiques.

LEDUC et DUFour [5] furent done conduits à utiliser une autre méthode de chauffage par courant haute fréquence, en employant cette fois non plus des champs magnétiques variables, mais au contraire des champs électriques à très haute fréquence, et en bénéficiant des phénomènes qui prennent naissance dans les produits cette fois non conducteurs, lorsque ceux-ci sont soumis à ces champs électriques variables.

On a fourni plusieurs théories pour expliquer ces phénomènes qui se manifestent par un échauffement parfois très brutal si les conditions opératoires sont choisies convenablement. La théorie la plus souvent admise est celle de Dеву d'après laquelle, lorsque les molécules polaires sont placées dans un champ électrique, ces molécules qui se comportent à la façon d'un doublet, c'est-à-dire d'un système de deux sphères électrisées portant des charges égales et de signe contraire se trouvent soumises à des inversions continuelles de $180^{\circ}$, lorsqu'à chaque fois l'extrémité positive du doublet est attirée par l'armaturè négative qui crée le champ électrique. Comme cette armature change de signe un nombre considérable de fois par seconde, les doublets tendent à se retourner sur eux-mêmes, mais leur mouvement est freiné par la viscosité du milieu et l'énergie dépensée par le freinage se retrouve sous forme de chaleur engendrée dans le milieu qui contient ces doublets.

On a pu établir [6] une formule qui donne l'énergie transformée en chaleur lorsqu'un corps non conducteur est placé entre deux armatures entre lesquelles on fait varier un grand nombre de fois la différence de potentiel. Cette formule est la suivante : 


$$
\mathrm{W}=\mathrm{E}^{2} \cdot 2 \pi . \mathrm{f} . \mathrm{C} \cdot \operatorname{tg} \delta
$$

dans laquelle :

W est l'énergie dissipée dans le milieu en watts ;

E la tension efficace entre les électrodes;

$f$ la fréquence du courant ;

C la capacité du condensateur constitué par les deux armatures entre lesquelles est placé le produit à chauffer ;

$\operatorname{tg} \delta$ le facteur de puissance de ce dernier.

Cette formule ou d'autres parfois plus simples qui en découlent nous permettent de définir les facteurs qui vont agir sur l'échauffement. On voit par exemple que pour augmenter l'énergie dissipée dans le corps à chauffer, il y a intérêt à utiliser la fréquence la plus élevée possible. Il est parfois nécessaire d'ailleurs d'adopter un compromis, lorsqu'en particulier l'objet à traiter à de trop grandes dimensions, ce qui pourrait entraîner une distribution irrégulière du champ électrique. D'autre part, plus élevée est la fréquence, plus onéreux en général est le courant produit à cette haute fréquence et le chauffage électronique n'a d'ailleurs pu trouver les applications qu'il rencontre aujourd'hui que le jour où l'on est parvenu à prodnire dans des conditions économiques des courants à très haute fréquence, grâce à la technique acquise par les spécialistes dans le domaine des radio-communications.

L'équation qui précède montre également que l'énergie transformée en chaleur est proportionnelle au carré de la tension efficace entre électrodes. Toutefois dans la pratique, il est difficile de travailler à des tensions trop élevées, non pas que l'on ignore produire ces tensions, mais parce qu'il devient nécessaire de prendre des précautions très complexes lorsqu'on travaille avec des tensions trop élevées.

Enfin, l'équation montre également que l'énergie dissipée dépend du facteur de puissance du produit, mais là on ne peut le modifier à volonté, puisque le produit à chauffer doit être en général traité tel qu'il est. Finalement, c'est également la capacité du condensateur constitué par l'installation qui fixera aussi la vitesse d'échauffement et, dans ce cas, ce sont les dimensions données à l'appareil qui interviendront en dernier lieu.

L'emploi du courant électrique pour le chauffage a toujours été considéré comme une opération onéreuse. L'emploi du chauffage électronique alimenté en courant à haute fréquence, est encore plus cher que le chauffage électrique ordinaire, puisque d'une part, il nécessite l'achat d'une installation productrice de courant qui représente des dépenses élevées et que d'autre part, la transforma- 
tion du courant à fréquence normale, en courant à haute fréquence, est également une opération coûteuse.

Les progrès qui ont été réalisés au cours de cés dernières années dans les pays anglo-saxons ont entraîné des modifications profondes de l'outillage de production des courants haute fréquence. Les appareils sont devenus beaucoup plus imposants, leur puissance a été augmentée et il a été conçu de nombreux dispositifs accessoires qui en rendent le fonctionnement plus sûr et en réduisent les dangers. On peut utiliser deux groupes d'appareils pour la production des courants haute fréquence, soit des machines rotatives, soit des générateurs électroniques. Les seconds sont d'ailleurs rarement employés lorsqu'on désire produire des fréquences inférieures à 100.000 périodes par seconde, et ce sont donc les appareils comportant des lampes d'émission que l'on utilise pour la production des courants servant au chauffage électronique.

\section{Applications du chauffage électronique à la stérilisation des liquides}

Une des premières applications que l'on ait envisagée pous l'emploi du chauffage électronique à la stérilisation des liquider a été la pasteurisation de la bière. Cette étude a en particulierété entreprise par Trwos et plusieurs de ses Collaborateurs [7], l'opération portant sur la bière en bouteille. L'appareil comportait une chaine transporteuse qui obligeait les bouteilles à circuler entre des électrodes. On utilisait une fréquence variable suivant les essais de 15 à 210 mégacycles, une tension comprise entre 7 à 10 kilovolts et la vitesse de déplacement de la bande transporteuse était réglée de telle sorte que les bouteilles se trouvaient soumises à l'action du champ électrique pendant une durée de 5 à 10 sec ondes. Ces essais ont été également répétés sur le cidre, sur les jus de fruits, sur le lait et il semble que les premières recherches n'aient pas donné d'excellents résultats.

La Radio Corporation of America qui s'est spécialisée dans la construction du matériel produeteur de courant haute fréquence utilisé dans le chauffage diélectrique, a alors repris la question s sus une autre forme. Le liquide à pasteuriser, par exemple le lait, circule à l'intérieur d'un conduit en verre ou en une autre matière électrique et l'on crée à l'intérieur de ce conduit un champ électrique variant à très haute fréquence, de façon à échauffer très rapidement le liquide qui cireule dans le conduit. En adoptant cette technique, on s'est alors heurté à des difficultés d'ordre mécaniques, dues à ce qu'il est très difficile de réaliser dans ces conditions un écoulement parfaitement réguliè du liquide à l'intérieur du conduit. Le liquide, en effet, au cours de son déplacement, vient 
frotter sur les parois du conduit. Il en résulte que la partie du fluide qui circule le long du conduit a une vitesse de déplacement nettement inférieure, en raison $d u$ frottement, à celle de la partie du fluide circulant au centre. Dans ces conditions, les parties du fluide qui sont retardées par le frottement restent soumises à l'action du champ électrique pendant un temps sensiblement plus long que les autres et il en résulte qu'elles doivent subir une surchauffe si les conditions du chauffage ont été calculées par exemple pour assurer une pasteurisation satisfaisante du liquide circulant au centre du conduit.

Si cette méthode de chauffage est appliquée au lait, ce dernier par suite de ces phénomènes de surchauffe peut présenter un goût de brûlé ; lorsqu'on opère sur d'autres produits, comme par exemple sur des jus de fruits, il peut se former des dépôts de matière qui forment un enduit adhérent sur les parois intérieures du conduit et qui aggravent encore les risques de surchauffe.

C'est pour éviter cet inconvénient que la Radio Corporation of America [8] a imaginé un dispositif dans lequel le courant de matières fluides ne circule plus entre les éleetrodes à l'intérieur d'un conduit, mais se déplace à l'état libre sous forme d'un jet dont on peut estimer que toutes les particules ont unè vitesse uniforme dans toute la section du jet. Dans ce cas, en effet, le liquide extérieur subit simplement un retard négligeable dû au frottement produit par

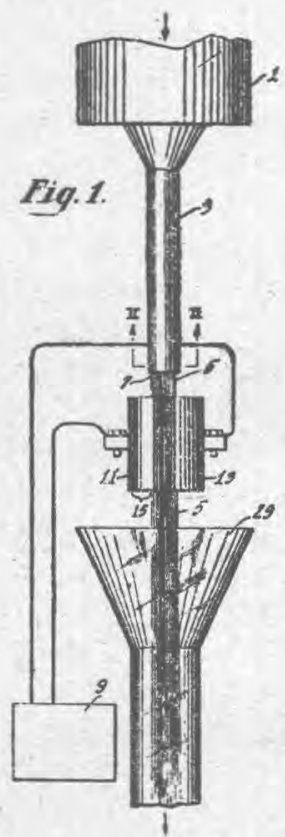

l'air ou par le milieu a mbiant gazeux qui entoure le liquide en mouvement. D'autres dispositifs accessoires sont également prévus pour réduire au minimum la possibilité de turbulence dans le courant de liquide en mouvement.

La figure 1 représente une des formes. de l'appareil créé par la firme américaine. 1 est un réservoir contenant le liquide à traiter, 3 est une tuyère de section transversale circulaire, qui permet de produire l'écoulement de la matière fluide sous la forme d'un jet continu 5. La vitesse d'écoulement du jet est pratiquement déterminée par la pression exercée sur la colonne de liquide dans les tuyaux, par la masse de liquide eontenue dans le réservoir. On peut également prévoir une pompe auxiliaire pour régler la vitesse du jet. Pour réduire en outre toute turbulence dans le courant de liquide, on fait passer celui-ci à travers une surface ajourée constituée par exemple par un tamis métallique placéà l'extrémité du tuyau. 
Les électrodes 11 et 13 peuvent être constituées par une paire de plaques métalliques courbes, les surfaces intérieures concaves de ces plaques étant à une certaine distance du jet de liquide.

\section{Application du chauffage diélectrique à la pasteurisation du lait}

On sait que dans le procédé habituel de pasteurisation basse, le lait est chauffé jusqu'à une température de $61-62^{\circ}$ et maintenu à cette température pendant environ 30 minutes. Dans ces conditions, sa teneur en bactéries est habituellement réduite jusqu'à $1 \%$ environ de sa valeur initiale. Si le temps de chauffage est réduit, la teneur en bactéries est notablement plus grande, s'il est plus long, le lait risque d'acquérir un goût de euit et le volume apparent de erème qu'il fournit est diminué. Si par ce procédé de pasteurisation, la plupart des bactéries pathogènes sont détruites, il n'en est pas de même pour d'autres et par exemple cette température de pasteurisation correspond à peu près à la température optimum pour le développement de certaines bactéries thermophiles, tandis que les bactéries thermoduriques survivent très bien au traitement.

C'est pour cela que ce procédé est souvent remplacé par la pasteurisation haute qui consiste à maintenir le lait pendant un temps plus court à des températures de 71-720. Le traitement peut s'effectuer par exemple en faisant passer le lait en couche mince sur des plaques chauffées et en le refroidissant ensuite rapidement. On a cherché à utiliser le chauffage électrique pour réaliser cette opération, en faisant passer le lait entre deux électrodes. Comme ce liquide présente une certaine conductibilité, il est traversé par le courant amené par les électrodes et par l'effet Joule il se trouve échauffé. Mais si l'on emploie des électrodes en carbone, celles-ci sont poreuses et retiennent une certaine quantité de lait, en même temps qu'il y a passage dans le liquide de petites particules de matières qui peuvent en modifier le goût; si les électrodes sont en acier inoxydable on en argent, il peut y avoir assombrissement de la couleur du lait.

On a donc eherché, pour assurer un chauffage instantané du lait sans contact avec les électrodes, à appliquer le procédé de chauffage par radiofréquence que nous avons décrit plus haut. Les essais entrepris par la Radio Corporation of America [9] ont montré qu'en élevant la température du lait à une valeur comprise entre 90 et son point d'ébullition et en soumettant le lait à cette température pendant une fraction de seconde seulement, on parvient à réaliser une pasteurisation complète, à condition d'assurer un refroidissement extrêmement rapide du lait. 
Ce procédé a en outre l'avantage de peu modifier le goût du lait et le volume apparent de crème qu'il fournit. Pour qu'en particulier, cette dernière condition soit remplie, il est nécessaire d'assurer le refroidissement rapide du lait et surtout d'atteindre dans le plus court délai possible une température inférieure à $57^{\circ} \mathrm{C}$. L'expérience en effet a permis d'établir la règle générale suivante : plus la température de pasteurisation est élevée, plus grande est la quantité de chaleur qui doit être enlevée pour obtenir un volume apparent élevé de crème. Si par exemple le lait a été soumis à une température de pasteurisation de $96^{\circ}$, puis refroidi ensuite à une température d'environ $30^{\circ} \mathrm{C}$., le tout en une période de temps de moins de une seconde, le volume apparent de crème n'est pas modifié, tandis que si l'on se contente de refroidir le lait à $47^{\circ}$ seulement, ce volume de crème peut être réduit, ce qui est un inconvénient au point de vue commercial, lorsque le lait non homogénéisé est mis sur le marché en bouteilles de verre.

Pratiquement, étant donné, comme nous l'avons indiqué précédemment, le prix élevé des caloriès fournies par le courant haute fréquence, on ne cherche pas à élever le lait à la température de $96^{\circ}$ uniquement par chauffage diélectrique. On commence par le chauf-

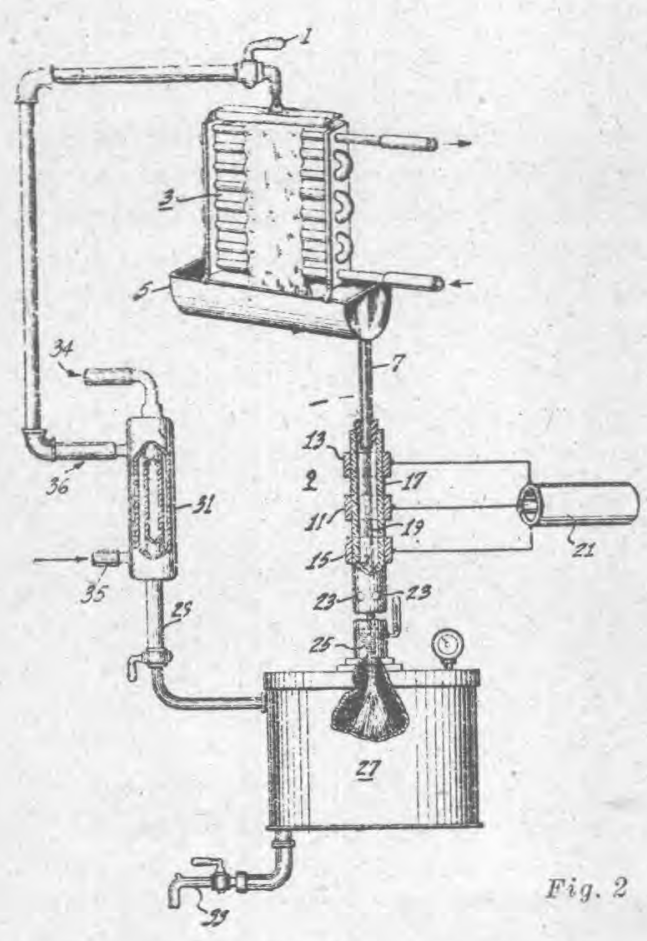

fer à la température de $60^{\circ}$ par un moyen courant, et ce n'est qu'alors qu'on le soumet à l'action du champ électrique à haute fréquence.

La figure 2 représente une installation de pasteurisation par haute fréquence répondant aux conditions que nous venons de décrire. Le lait provenant du robinet $\mathrm{n}^{0} 1$ s'écoule tout d'abord sur un serpentin parcouru par un courant d'eaú chaude et de là est dirigé dans un appareil de traitement à haute fréquence représenté en 9. Dans le cas de la figure, le champ électrique est créé par 3 paires d'anneaux disposés les uns au-dessus 
des autres et à l'intérieur desquels le lait circule sous forme d'un jet de section eirculaire. L'énergie fournie aux électrodes doit être suffisamment élevée pour chauffer instantanément le lait à la te mpérature de $96^{\circ}$.

Après son passage à l'intérieur des électrodes, le lait est recueilli dans un réservoir 27 au moyen d'un entonnoir 25 qui, étant continuellement rempli de liquide, sert de joint étanche entre le système de refroidissement à l'intérieur duquel existe le vide et le conduit à l'intérieur duquel circule le lait. .

Le dispositif de refroidissement peut être d'un type très clasr sique, l'élimination des calories étant assurée en grande partie pa-

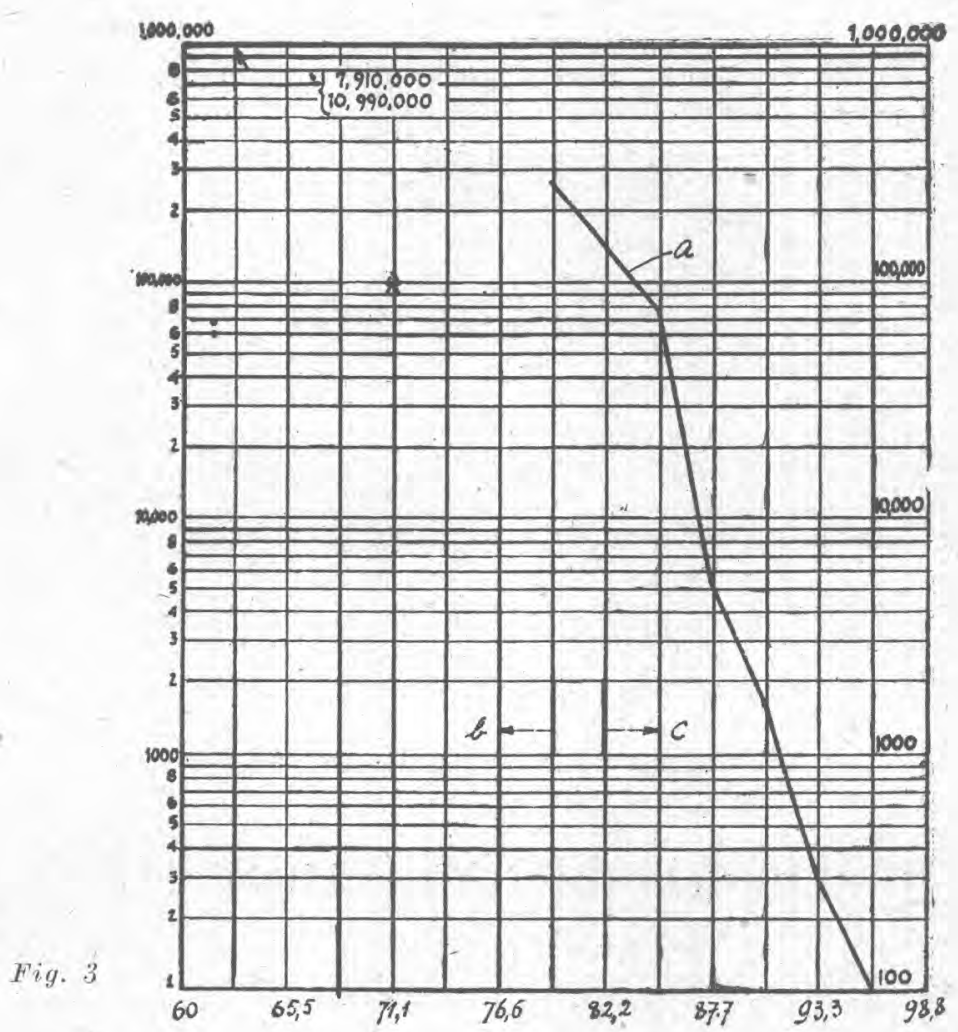

suite de la vaporisation d'une certaine fraction de l'eau que contient le lait. Cette eau est ensuite condensée dans un condenseur refroidi par le lait brut avant son passage dans l'appareil de pasteurisation et ajoutée au lait après refroidissement.

II est intéressant de donner quelques chiffres concernant le fonctionnement électrique d'une telle installation; l'expérience a 
montré d'ailleurs que les données expérimentales sont très voisines de celles que permet de calculer la théorie. Si partant de lait porté à $60^{\circ}$, on élève cette température à $96^{\circ}$ par chauffage par radiofréquence, il faut fournir à l'installation une énergie de 2.096 watts-minute par litre de lait. On voit qu'un kilowatt permet d'effectuer la pasteurisation de 28 1. 6 de lait à l'heure et qu'un oscillateur d'une puissance de 100 kilowatts, ce qui est un appareil d'une construction courante aujourd'hui, est capable d'assurer la pasteurisation de 2.860 litres de lait à l'heure.

Dans la figure 3 , nous avons reproduit une courbe qui montre au moyen d'une échelle logarithmique la variation du nombre de bactéries contenues dans le lait en fonction de la température de pasteurisation. On voit combien une élévation de cette dernière permet de réduire dans des proportions énormes le nombre de bactéries de tous types existant dans le lait.

\section{RÉFÉRENCES}

[1] M. Deníń́né. Le Lait, t. XXIII, p. $289,1943$.

[2] L. V. Burton. Elect. Contracting, t. XLIV, no 1, p. 59, 1945.

[3] J. A. Reavelt, Ind. Chemist, t. XX, no 229, p. 54, 1944.

[4] V. W. Sherman. Electronics, t. XVII, p. 94, 1944.

E. Rushton, E. C. Stanley et A. W. Seott. Chem. and Ind., t. XXXV, p. $274,1945$.

[5] Leduc et Dufour. Brevet français 808.612, 1937. Encyclopédie technologique du caoutchouc, t. II, p. 839,1938 .

[6] R. M. BAKER et C. J. MAdsen, Electr. Eing., t LXIV, no 2, p. 50, 1945.

[7] A. C. Titus. Am. Brewer, t. LXXIX, no 2, p. 23, p. 65, 1946.

L. E. Jones. Am. Brewer, t. LXXVIII, no 9, p. $21,1945$.

C. N. Sifben. Am. Brewer, t. LXXVIII, no 11, p. 26, 1945.

F. W. Wenmiler. A m. Brewer, t. LXXVIII, no 11, p. 25, 1945.

[8] Radio Corporation of America. Brevet français 915.115, du 27 sep. tembre 1945.

[9] Radio Corporation of America. Brevet français 915.114 du 27 septembre 1945 .

\section{BIBLIOGRAPHIE ANALYTIQUE}

\section{LES LIVRES}

Cooper (Herbert J.). - Scientific instruments (Instruments scientifiques). 1 vol. relié toile de 305 pages, avec nombreuses illustrations. Chemical Publishing Co., Ine. Brooklyn, NewYork, 1946. Prix : 6 dollars.

L'A. passe en revue une grande quantité d'instruments de mesures physiques, non seulement d'instruments de laboratoire, mais aussi de ceux utilisés dans l'industrie et le commerce. Les instruments sont décrits avec détails et une large place est faite à l'examen des principes sur lesquels ils sont basés ainsi qu'aux méthodes de mesure. 